\title{
Resultados del Programa Nacional de Implantes Cocleares: Servicio de Otorrinolaringología del Hospital Barros Luco Trudeau
}

\author{
Results of the National Cochlear Implant Program: ENT Department \\ Barros Luco Trudeau Hospital
}

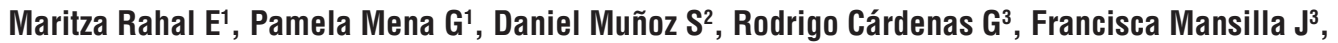 \\ Felipe Cardemil M ${ }^{1,4}$.
}

\begin{abstract}
RESUMEN
Introducción: La hipoacusia sensorioneural bilateral profunda es la tercera causa de discapacidad en Chile, con una incidencia de entre 1 a 3 casos por cada 1.000 nacidos vivos, siendo muchos casos susceptibles de ser tratados mediante implante coclear.

objetivo: Describir resultados de la cohorte de pacientes implantados en el Hospital Barros Luco Trudeau.

Material y método: Estudio de cohorte retrospectiva. Se reportan los resultados de la totalidad de pacientes implantados en el período 2003-2011.

Resultados: Fueron implantados 80 pacientes. La mediana de edad de implantación fue 48 meses. Treinta y siete $(46,25 \%)$ pacientes fueron mujeres, $7(8,75 \%)$ fueron prematuros, y $14(17,5 \%)$ tenían el antecedente de meningitis bacteriana. Sesenta y ocho (85\%) fueron diagnósticos prelinguales, $9(11,25 \%)$ poslinguales, y $3(3,75 \%)$ perilinguales. EI PTP promedio prequirúrgico fue 111,82 dB, y el posquirúrgico con implante 25,36 dB ( $p$ $<0,0001$ ). Respecto a integración, el $100 \%$ de los pacientes poslinguales, el $67,7 \%$ de los prelinguales, y el $100 \%$ de los perilinguales se encuentran integrados a sus actividades. Se ha observado el 5\% de complicaciones: una parálisis facial, un hematoma infectado y dos alergias a cera de hueso.

Conclusión: Los resultados de este programa son altamente satisfactorios. Todos los pacientes se encuentran en seguimiento hasta la fecha con una buena adherencia terapéutica y rehabilitación. La evaluación de este programa permite plantear que debería ampliarse.
\end{abstract}

Palabras clave: Hipoacusia sensorioneural bilateral profunda, implante coclear, política pública de salud.

\footnotetext{
${ }^{1}$ Médico, Servicio de Otorrinolaringología, Hospital Barros Luco Trudeau - Facultad de Medicina, Universidad de Chile.

${ }^{2}$ Médico, Magíster en Bioestadística, Universidad de Chile.

${ }^{3}$ Fonoaudiólogo, Servicio de Otorrinolaringología, Hospital Barros Luco Trudeau.

${ }^{4}$ Programa de Doctorado en Salud Pública, Escuela de Salud Pública, Universidad de Chile.
} 


\section{ABSTRACT}

Introduction: Profound bilateral sensorioneural hearing loss is the third leading cause of disability in Chile, with an incidence of 1 to 3 cases for every 1.000 born, which may be managed with cochlear implants.

Aim: Describe results of the cohort of patients implanted at Barros Luco Trudeau Hospital.

Material and method: Retrospective cohort study. We report the results of all patients implanted in the period from 2003 to 2011.

Results: 80 patients were implanted. The median age at implantation was 48 months. $37(46.25 \%)$ patients were female, $7(8.75 \%)$ were premature, and $14(17.5 \%)$ had a history of bacterial meningitis. 68 (85\%) were prelingual diagnosed, $9(11.25 \%)$ postlingual, and $3(3.75 \%)$ perilinguals. The average preoperative PTP was $111.82 \mathrm{~dB}$, and postoperative was $25.36 \mathrm{~dB}(p<0.0001)$. Regarding integration, $100 \%$ of postlingual patients, $67.7 \%$ of prelingual, and $100 \%$ of perilinguales are fully integrated into their activities. There have been $5 \%$ complications: one facial paralysis, one infected hematoma, and two bone wax allergies.

Conclusion: The results of this program are highly satisfactory. All patients are up to date with good adherence and rehabilitation. The evaluation of this program allows us to suggest that should be expanded.

Key words: Bilateral profound sensorineural hearing loss, cochlear implant, health public policy.

\section{INTRODUCCIÓN}

La hipoacusia es una importante causa de discapacidad en Chile, ocupando el tercer lugar en frecuencia ${ }^{1}$. La hipoacusia sensorioneural bilateral es la discapacidad congénita más frecuente, con una incidencia general estimada que oscila entre 1 y 3 casos de entre 1.000 nacidos vivos ${ }^{2}$. En Chile, un estudio retrospectivo que incluyó a más de 10.000 recién nacidos tamizados entre los años 2001 y 2007 reportó una tasa de 2,8 por cada 1.000 recién nacidos ${ }^{3}$. Esta enfermedad genera importantes consecuencias. A nivel individual afecta la adquisición de lenguaje, retrasa el aprendizaje, disminuye las habilidades cognitivas, se asocia a problemas de comportamiento y menor bienestar psicológico y disminuye la productividad laboral ${ }^{4}$. Asimismo, los padres, familiares o cuidadores de niños hipoacúsicos presentan un importante estrés físico-psicológico ${ }^{5}$. Por lo tanto, tiene una importante carga de enfermedad asociada.

Como sus etiologías son múltiples y muchas de ellas no prevenibles, una intervención costoefectiva es la implementación auditiva de estos pacientes, particularmente el implante coclear, que ha demostrado excelentes resultados internacionales ${ }^{6-10}$. Dentro de éstos, cabe mencionar la mejor adquisición de lenguaje oral, adecuada inclusión social y mejor calidad de vida de los pacientes implantados, resultados que son aún mayores mientras más precoz se realice la implementación ${ }^{11,12}$. Además de esto, el implante coclear estimula directamente el nervio auditivo y el cerebro, evitando muchos de los efectos nocivos de la deprivación sensorial, y contribuyendo al desarrollo de neuroplasticidad $^{12}$. A largo plazo, los resultados son también altamente satisfactorios, comparable con niños normales, y siendo estables en el transcurso de los años, no identificándose deterioro en el tiempo ${ }^{13}$. De esto se desprende la relevancia de instaurar un programa tamizaje universal para la detección temprana de hipoacusia congénita ${ }^{14,15}$, recurso que aún no se encuentra disponible en Chil ${ }^{16}$, aun cuando está sólidamente establecida la factibilidad y conveniencia de hacerlo. La estrategia de realizar un tamizaje auditivo sólo en pacientes con factores de riesgo ha demostrado ser insuficiente, ya que cerca de la mitad de los pacientes con hipoacusia congénita no presentan ningún factor de riesgo clínicamente aparente ${ }^{17}$. 
Desde un punto de vista económico, el implante coclear corresponde a una intervención costosa, pero cuyos resultados sobrepasan con creces la inversión realizada ${ }^{10,18}$, disminuyendo tanto los gastos personales, como los años de vida saludables perdidos (AVISA) relacionados con la hipoacusia. Con el implante coclear se logra un desarrollo de lenguaje oral en el paciente, facilitando la asistencia a una institución educativa regular. En consecuencia, los costos en educación son menores comparados con los que requieren los individuos con otro tipo de ayudas auditivas. Además de generar un ahorro económico, este dispositivo mejora el estado de salud del paciente, compensando así el valor de la intervención quirúrgica para implantarlo ${ }^{19}$. Según un estudio de Peñaranda y $\operatorname{col}^{20}$, el retorno de la inversión por cada dólar invertido en el implante coclear para tratar un paciente con hipoacusia sensorioneural es de US\$2,07, por lo que el implante coclear sería una alternativa de solución eficiente y efectiva para corregir la hipoacusia sensorioneural en los niños, dado que genera ganancias auditivas, comunicativas y económicas a los pacientes y sus familias ${ }^{21}$.

En Chile el año 2003 el Ministerio de Salud implementó un programa de implantes cocleares ${ }^{22}$, siendo uno de los centros de referencia de éste el Hospital Barros Luco Trudeau, centro hospitalario terciario de alta complejidad.

\section{OBJETIVO}

El objetivo del presente estudio es describir la cohorte de pacientes con hipoacusia sensorioneural bilateral profunda implantados en el Hospital Barros Luco Trudeau.

\section{MATERIAL Y MÉTODO}

Se realizó un estudio de cohorte retrospectiva que incluyó a la totalidad de pacientes implantados entre el período enero 2003 a diciembre 2011 en el Servicio de Otorrinolaringología del Hospital Barros Luco Trudeau, el que cuenta con un equipo de 7 otorrinolaringólogos especialistas con entrenamiento formal en implantes cocleares, y un equipo de fonoaudiólogos capacitados en calibración audiológica, rehabilitación y seguimiento de los pacientes con implante coclear. El estudio contó con aprobación del comité de ética del Hospital.
Se recolectaron datos epidemiológicos, variables que eventualmente influían en la rehabilitación auditiva (antecedentes mórbidos, etapa de diagnóstico, edad de implantación, pre y poslinguales), y resultados de la implementación tales como: promedio de promedio tonal puro (PTP) pre y posquirúrgico, integración escolar y laboral, percepción acústica del habla evaluada mediante la escala de Geers y Moog (siendo mejor la percepción acústica a mayor puntaje) y la presencia de complicaciones derivadas del procedimiento.

Los datos fueron analizados en el programa Stata 12.1 (StataCorp. 2011. CollegeStation, TX: StataCorp LP). Para variables cuantitativas se calcularon promedios y desviación estándar, o mediana y rango intercuartil (RIC) según correspondiera, y para variables categóricas se calcularon frecuencias absolutas y relativas. Para analizar diferencias entre variables cuantitativas en 2 categorías se utilizó $t$ de student 0 Mann Whitney según correspondiera, y cuando existían más de 2 categorías se utilizó Kruskall Wallis. Para todos los análisis se consideró significativo un valor de $p$ igual 0 menor a 0,05 .

\section{RESULTADOS}

Fueron implantados 80 pacientes con el diagnóstico de hipoacusia sensorioneural bilateral profunda. La mediana de edad de implantación del grupo total fue 48 (RIC 32,572) meses. Ocho de ellos fueron adultos al momento de la colocación del implante (10\%), y 37 pacientes fueron mujeres $(47,25 \%)$. Respecto a los antecedentes mórbidos, $23(28,75 \%)$ de ellos tenían al menos uno: 14 $(17,5 \%)$ tenían el antecedente de meningitis bacteriana, $7(8,75 \%)$ fueron pacientes prematuros, $3(3,75 \%)$ tenían parálisis cerebral, $2(2,5 \%)$ retraso del desarrollo sicomotor (RDSM), 1 (1,25\%) epilepsia y $1(1,25 \%)$ trastorno por déficit atencional con hiperactividad (TDAH) (Tabla 1). La mediana de edad de implantación del grupo de niños prematuros fue 35 (RIC 28-44) meses, mientras que la de los niños no prematuros fue de 43 (RIC 32-61) meses, no existiendo diferencia significativa entre estas edades $(p=0,08)$.

Respecto a la etapa del diagnóstico, 68 (85\%) fueron prelinguales, con una mediana de edad de implantación de 42 (RIC 29-60) meses, 9 (11,25\%) poslinguales, con una mediana de edad de implantación de 39 años (468 meses, RIC 156-600); y 3 (3,75\%) 
Tabla 1. Antecedentes mórbidos en la cohorte de pacientes sometidos a implante coclear, Hospital Barros Luco Trudeau, período 2003-2011

\begin{tabular}{|l|c|}
\hline Antecedentes mórbidos & Frecuencia \\
\hline Meningitis & $14(17,5 \%)$ \\
\hline Prematurez & $7(8,75 \%)$ \\
\hline Parálisis cerebral & $3(3,75 \%)$ \\
\hline RDSM & $2(2,5 \%)$ \\
\hline Epilepsia & $1(1,25 \%)$ \\
\hline TDAH & $1(1,25 \%)$ \\
\hline
\end{tabular}

RDSM: Retraso del desarrollo sicomotor.

TDAH: Trastorno de déficit atencional con hiperactividad.

perilinguales, con una mediana de edad de implantación de 48 (RIC 37-84) meses, existiendo diferencia significativa en la edad entre el grupo poslingual con los otros dos grupos $(p<0,0006)$. Al evaluar la etapa del diagnóstico según edad de aparición, se aprecia que la totalidad de los 7 pacientes prematuros fueron prelinguales, y de los 65 pacientes niños no prematuros, 3 fueron perilinguales (4,6\%), 3 fueron poslinguales $(4,6 \%)$, y 59 fueron prelinguales $(90,8 \%)$.

EI PTP promedio prequirúrgico sin implementación fue de $111,82 \mathrm{~dB}( \pm 9,5)$, y el posquirúrgico con implante $25,36 \mathrm{~dB}( \pm 12,9)(p<0,0001)$. Al analizar el PTP prequirúrgico según grupo de implantación, se aprecia que el grupo prelingual presentó un PTP de 113,05 ( \pm 8,5), el poslingual un PTP de $104,8( \pm 10,4)$, y el grupo perilingual un PTP de 107,7 $( \pm 19,7)$, existiendo una diferencia significativa entre el promedio del grupo prelingual $y$ el poslingual $(p=0,04)$. Al evaluar el PTP posquirúrgico según grupo de implantación, se aprecia que el grupo prelingual presentó un PTP de 26,3 $( \pm 13,7)$, el poslingual un PTP de $18,7( \pm 2,9)$, y el grupo perilingual un PTP de $20( \pm 4,4)$, sin apreciar diferencia significativa $(p=0,19)$. En el análisis dentro de cada grupo de implementación se obtuvo que para el grupo prelingual hubo una diferencia pre y posquirúrgica de $86,75 \mathrm{~dB}(\mathrm{p}<0,0001)$, para el grupo poslingual esta diferencia fue de $86,1 \mathrm{~dB}$ $(p<0,0001)$ y para el grupo perilingual dicha diferencia fue de $87,7 \mathrm{~dB}(p<0,0001)$. (Figura 1).

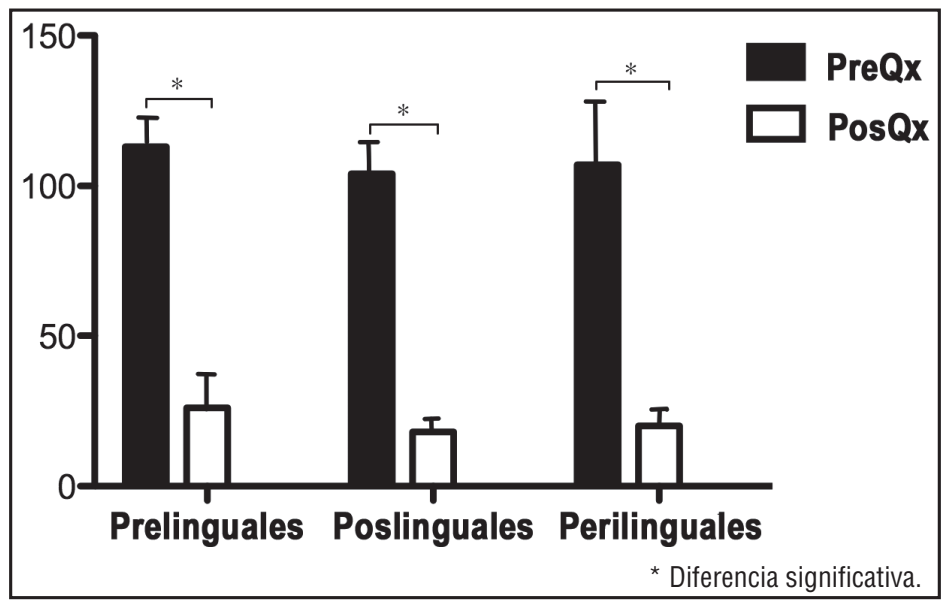

Figura 1. Promedios tonales puros pre y posimplante coclear según etapa de adquisición del lenguaje. 
Respecto a integración, la totalidad de los pacientes hipoacúsicos que fueron implantados en la etapa poslingual se encuentran integrados a sus actividades habituales. El $67,7 \%$ de los pacientes que fueron implantados prelingual se encuentran integrados y asistiendo a una escuela normal, con apoyo de fonoaudiólogo, y el $100 \%$ de los perilinguales se encuentra integrado a una escuela normal. No hubo diferencia significativa en la integración según el momento del implante $(p=0,06)$. No se apreció diferencia significativa en el nivel de PTP preimplante $(p=0,07)$ ni posterior al implante $(p=0,052)$ según la integración.

Dos de los pacientes presentaron neuropatía auditiva, diagnosticada con BERA plano y otoemisiones acústicas positivas. Uno correspondió a un paciente de sexo masculino, que fue el primer paciente implantado de la serie, y que se encuentra con un PTP en $40 \mathrm{~dB}$ asistiendo a escuela especial y con mala percepción acústica del habla (0 punto en escala de Geers y Moog); la segunda, es una niña que además presenta una agenesia del cuerpo calloso, fue evaluada con electrococleografía, con buen resultado en el lado derecho, por lo que se implantó ese lado, actualmente con escasa percepción acústica del habla (1 punto en escala de Geers y Moog) y escuchando en $50 \mathrm{~dB}$.

De la serie, se aprecia que hubo $14(17,5 \%)$ pacientes que tienen el antecedente de meningitis bacteriana. No se aprecia diferencia significativa en el PTP preimplante entre los pacientes con meningitis y los sin meningitis $(p=0,15)$. Sí se observa una diferencia significativa en el PTP posimplante, apreciándose en los pacientes sin meningitis un PTP de 23,6 $( \pm 9,5)$ y en con meningitis un PTP de 32,6 $( \pm 22)(p=0,01)$. Al realizar este análisis considerando sólo a los niños prelinguales, este resultado se repite, con un PTP en los sin antecedente de meningitis de $24,3( \pm 9,9)$ y en los con meningitis de $37,7( \pm 24,5)(p=0,003)$. Al evaluar el antecedente de meningitis y la integración, se aprecia que no existe asociación significativa entre estas dos variables ( $p=0,99$, y $p=0,717$ al considerar sólo pacientes prelinguales). Tampoco se encontró asociación significativa entre el antecendente de meningitis y la percepción acústica del habla, evaluada mediante la escala de Geers y Moog $(p=0,06)$. Estos datos apoyan el hecho que aunque los pacientes con meningitis presentan un PTP más alto, esto no impacta en su integración escolar ni en su percepción del habla.

Al evaluar la percepción acústica del habla mediante la escala de Geers y Moog, se aprecia que la mediana en la serie fue de 3 puntos (RIC 1-5). Al analizar el puntaje según integración escolar, se aprecia que los pacientes que presentan integración escolar tienen una mediana de 4 puntos (RIC 1-6), mientras que los que no se han integrado presentan una mediana de 1 (RIC 1-4) ( $p=0,001)$. Al repetir este análisis sólo en los pacientes prelinguales, se aprecia que los que presentan integración escolar tienen una mediana de 3.5 puntos (RIC 1-5), mientras que los que no se han integrado presentan una mediana de 1 (RIC 1-4) ( $p=0,008)$ (Figura 2).

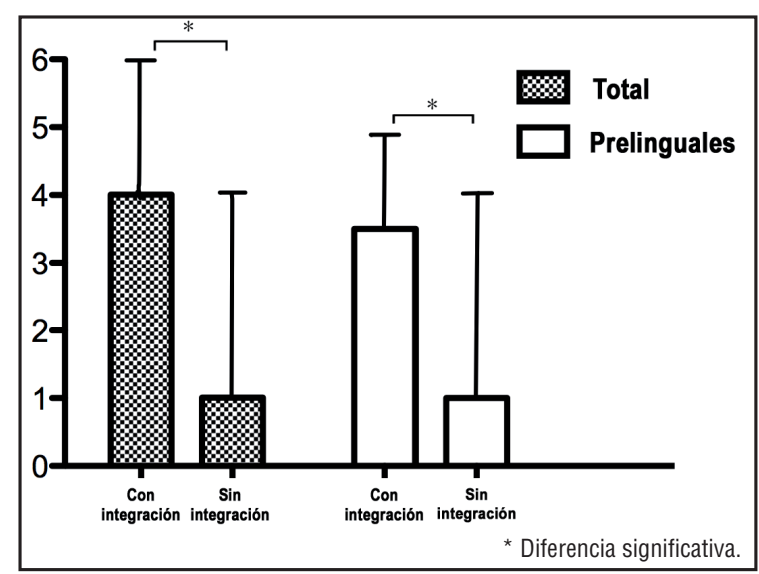

Figura 2. Puntajes de la escala de Geers y Moog de la muestra total y del subgrupo de pacientes prelinguales, de acuerdo a la integración escolar de los pacientes evaluados. 
En cuanto a las complicaciones, sólo se observaron cuatro ( $5 \%$ de los casos): un caso de parálisis facial recuperada, un hematoma que se infectó y que requirió cambio de implante, y dos casos de reacción alérgica a la cera de hueso y que también requirieron cambio de implante.

\section{DISCUSIÓN}

Los resultados de este programa son altamente satisfactorios, alcanzando una excelente proporción de rehabilitación auditiva, lo que ha llevado a un alto porcentaje de integración tanto escolar como laboral, siendo la percepción acústica del habla la variable más relacionada con aquello. Hasta la fecha todos los pacientes se encuentran en control audiológico con audiometría a campo libre y calibraciones, además del control fonoaudiológico. La coordinación con otros centros de rehabilitación y con escuelas que tienen proyecto de integración escolar han permitido una buena adherencia terapéutica y una mayor inclusión social. Aquellos pacientes que han demostrado menor progreso en su rehabilitación, tienen antecedentes mórbidos que lo explican, como parálisis cerebral 0 TDAH. Las complicaciones observadas se encuentran dentro de lo descrito en la literatura9 9 .

Cabe destacar el promedio de edad de implantación en los pacientes en etapa prelingual, de 4,4 años, que pone de manifiesto que el diagnóstico de hipoacusia sensorioneural en este grupo etario se está haciendo tardíamente, con las consecuencias conocidas que esto conlleva, situación que puede estar siendo favorecida por la falta de un screening universal de hipoacusia en nuestro país. En este sentido, este resultado resalta la necesidad de avanzar hacia la instalación de un programa de tamizaje universal nacional de hipoacusia.

La evaluación de este programa permite plantear que debería ampliarse, a fin de disponer de los recursos necesarios que permitan habilitar o rehabilitar a todos los pacientes usuarios del sistema público de salud que lo requieran, de manera de disminuir la brecha de inequidad existente. En este sentido, la promulgación de la nueva guía clínica AUGE de "Tratamiento de la hipoacusia moderada y severa en menores de 2 años" viene a regular la habilitación auditiva a los niños que lo requieran ${ }^{23}$. Sin embargo, queda pendiente aún avanzar hacia un programa de tamizaje universal que permita detectar los casos a tiempo, y legislar respecto a la necesidad de apoyo auditivo en los casos de hipoacusia hereditaria de aparición tardía 0 de otros casos que requieren implantes cocleares que no fueron diagnosticados antes de los 2 años de vida, sobre todo considerando la mayor edad promedio de diagnóstico en casos de ausencia de tamizaje universal².

\section{CONCLUSIÓN}

Es necesario ampliar los programas de implantes cocleares y la capacitación de los profesionales que trabajan con pacientes con hipoacusia, lo que debe ir de la mano con la instalación de programas de tamizaje universal en recién nacidos, considerando la relevancia que cobra la hipoacusia sensorioneural desde el punto de vista de salud pública, ya que es una causa de discapacidad frecuente, que tiene gran impacto a nivel individual y poblacional, y que es factible de tratar, con resultados satisfactorios y buena integración social, escolar y laboral de los afectados. Se hace imprescindible a la luz de estos resultados implementar un programa de detención e intervención precoz de hipoacusia, a fin de lograr habilitar y rehabilitar a estos pacientes a una edad más temprana, lo que permitiría mejorar aún más estos indicadores.

\section{Agradecimientos}

Los autores desean reconocer el dedicado trabajo del equipo de otorrinolaringólogos y fonoaudiólogos que participan del programa de implantes cocleares en el Hospital Barros Luco Trudeau, Dres. Ana María Contador C., Claudia Dentone S., Patricia Esquivel C., Úrsula Zelada B., Rodrigo Arregui V. y Rocco Calabrese G., y Fonoaudiólogos Rocío Carvajal D., Rose Fromin P., e Iván Villalobos A.

\section{BIBLIOGRAFÍA}

1. Encuesta CASEN, 2009. Disponible en http://www. ministeriodesarrollosocial.gob.cl/casen2009/ [Revisado el 20 de marzo de 2013].

2. Morton CC, Nance WE. Newborn hearing screening: a silent revolution. N Engl J Med 2006; 354: 2151-64. 
3. Nazar G, Goycoolea M, Godoy JM, Ried E, Sierra M. Evaluación auditiva neonatal universal: Revisión de 10.000 pacientes estudiados. Rev Otorrinolaringol Cir Cabeza Cuello 2009; 69: 93-102.

4. Estudio Nacional de la Discapacidad (ENDISC) 2004. http://www.ine.cl/canales/chile_estadistico/ encuestas_discapacidad/discapacidad.php [Revisado el 20 de marzo de 2013].

5. Meinzen-Derr J, Lim LH, Choo Di, Buyniski S, WILEY S. Pediatric hearing impairment caregiver experience: impact of duration of hearing loss on parental stress. Int J Pediatr Otorhinolaryngol 2008; 72(11): 1693-703.

6. Geers AE. Speech, Language, and Reading Skills After Early Cochlear Implantation. Arch Otolaryngol Head Neck Surg 2004; 130: 634-8.

7. Goycoolea M. y el Grupo Latinoamericano de Implante Coclear. Experiencia latinoamericana con el implante coclear. Rev Otorrinolaringol Cir Cabeza Cuello 2005; 65: 165-72.

8. Bond M, Mealing S, Anderson R, Elston J, Weiner G, TAYLOR RS, ET AL. The effectiveness and costeffectiveness of cochlear implants for severe to profound deafness in children and adults: a systematic review and economic model. Health Technol Assess 2009; 13(44).

9. Yoon PJ. Pediatric cochlear implantation. Curr Opin Pediatr 2011; 23 (3): 346-50.

10. Manrique M, Ramos A, Morera C, Cenjor C, Lavilla MJ, Boleas MS, Cervera-Paz FJ. Evaluación del implante coclear como técnica de tratamiento de la hipoacusia profunda en pacientes pre y poslocutivos. Acta Otorrinolaringol Esp 2006; 57 (1): 2-23.

11. May-Mederake B. Early intervention and assessment of speech and language development in young children with cochlear implants. Int $J$ Pediatr Otorhinolaryngol 2012; doi:10.1016/j. ijporl.2012.02.051.

12. Kral A, Sharma A. Developmental neuroplasticity after cochlear implantation. Trends Neurosci 2012; 35 (2): 111-22.

13. Peixoto MC, Spratley J, Oliveira G, Martins J, Bastos J, Ribeiro C. Effectiveness of cochlear implants in children: Long term results. Int $J$
Pediatr Otorhinolaryngol 2013 Jan 2. [Epub ahead of print].

14. Martin Ptok. Early Detection of Hearing Impairment in Newborns and Infants. Dtsch Arztebl Int 2011; 108(25): 426-31.

15. Wolff R, Hommerich J, Riemsma R, Antes G, Lange $S$, KLEIJNEN J. Hearing screening in newborns: systematic review of accuracy, effectiveness, and effects of interventions after screening. Arch Dis Child 2010; 95(2): 130-5.

16. Cornejo V, Raimann E, Cabello Jf, Valiente A, Becerra C, Opazo M et al. Past, present and future of newborn screening in Chile. J Inherit Metab Dis 2010; Aug 4. [En prensa].

17. Smith RJ, Bale JF, White KR. Sensorineural hearing loss in children. Lancet 2005; 365(9462): 879-90.

18. Monteiro E, Shipp D, Chen J, Nedzelski J, Lin V. Cochlear Implantation: A Personal and Societal Economic Perspective Examining the Effects of Cochlear Implantation on Personal Income. J Otolaryngol Head Neck Surg 2012; 41 Suppl 1: 43-8.

19. O'Nelll C, O'Donoghue GM, Archbold SM, Normand C. A cost-utility analysis of pediatric cochlear implantation. Laryngoscope 2000; 110(1): 156-60.

20. Peñaranda A, Mendieta JC, Perdomo Ja, Aparicio ML, Marín LM, García JM et al. Beneficios económicos del implante coclear para la hipoacusia sensorineural profunda. Rev Panam Salud Pública 2012; 31(4): 325-31.

21. Hutton J, Politi C, Seeger T. Cost-effectiveness of cochlear implantation of children. In Cochlear Implants in Children, Uziela, Mondarin M (eds). Adv Otorhinolaryngol 1995; 50: 201-6.

22. Guía de Práctica Clínica "Implante Coclear", Ministerio de Salud, Gobierno de Chile, 2008. Disponible en http://www.minsal.gob.cl/portal/ url/item/b157ccdf54b77a48e04001011e012f6b. pdf [Revisado el 20 de marzo de 2013].

23. Guía Clínica AUGE "Tratamiento de la hipoacusia moderada y severa en menores de 2 años", Ministerio de Salud, Gobierno de Chile, 2013. Disponible en http://www.minsal.cl/portal/url/ item/de429df07a91ca3ce040010165017ea0.pdf [Revisado el 8 de Julio del 2013]. 